\title{
INCREASED BRAIN LACTATE IS CENTRAL TO THE DEVELOPMENT OF BRAIN EDEMA IN RATS WITH CHRONIC LIVER DISEASE
}

\section{Cristina R. Bosoi, Claudia Zwingmann, Helen Marin, Christian Parent-Robitaille, Jimmy Huynh, Mélanie Tremblay, Christopher F. Rose}

\author{
Neuroscience Research Unit, Hôpital Saint-Luc (CRCHUM), Université de Montréal, Québec, Canada
}

Received 25 February 2013, Revised 4 October 2013, Accepted 11 October 2013, Available online 26 October 2013

\section{ABSTRACT}

Background \& Aims The pathogenesis of brain edema in patients with chronic liver disease (CLD) and minimal hepatic encephalopathy (HE) remains undefined. This study evaluated the role of brain lactate, glutamine and organic osmolytes, including myo-inositol and taurine, in the development of brain edema in a rat model of cirrhosis.

Methods Six-week bile-duct ligated (BDL) rats were injected with ${ }^{13} \mathrm{C}$-glucose and de novo synthesis of lactate, and glutamine in the brain was quantified using ${ }^{13} \mathrm{C}$ nuclear magnetic resonance spectroscopy (NMR). Total brain lactate, glutamine, and osmolytes were measured using ${ }^{1} \mathrm{H}$ NMR or high performance liquid chromatography. To further define the interplay between lactate, glutamine and brain edema, BDL rats were treated with AST-120 (engineered activated carbon microspheres) and dichloroacetate (DCA: lactate synthesis inhibitor).

Results Significant increases in de novo synthesis of lactate (1.6-fold, $p<0.001)$ and glutamine $(2.2$-fold, $p<0.01)$ were demonstrated in the brains of BDL rats vs. SHAM-operated controls. Moreover, a decrease in cerebral myo-inositol $(p<0.001)$, with no change in taurine, was found in the presence of brain edema in BDL rats $v s$. controls. BDL rats treated with either AST-120 or DCA showed attenuation in brain edema and brain lactate. These two treatments did not lead to similar reductions in brain glutamine.

Conclusions Increased brain lactate, and not glutamine, is a primary player in the pathogenesis of brain edema in CLD. In addition, alterations in the osmoregulatory response may also be contributing factors. Our results suggest that inhibiting lactate synthesis is a new potential target for the treatment of HE.

\section{Abbreviations}

HE, hepatic encephalopathy; MHE, minimal hepatic encephalopathy; CLD, chronic liver disease; TCA cycle, tricarboxylic acid cycle; NMR, nuclear magnetic resonance; BDL, bile-duct ligation; DCA, dichloroacetate; PDH, pyruvate dehydrogenase; PC, pyruvate carboxylase

\section{Keywords}

Hepatic encephalopathy; Nuclear magnetic resonance; Brain edema; Bile-duct ligation; Lactate; Glutamine

Bosoi, C.R. et al., 2014. Increased brain lactate is central to the development of brain edema in rats with chronic liver disease. Journal of Hepatology, 60(3), p.554-560. 


\section{INTRODUCTION}

Hepatic encephalopathy (HE) is a neuropsychiatric disorder, a major complication of both acute liver failure and chronic liver disease (CLD). Brain edema is a neuropathological feature of HE that contributes to intracranial hypertension (a fatal complication of acute liver failure, [1]); but is also associated with minimal HE (MHE) and CLD [2], [3], [4] and [5]. Characterized by impairment in concentration, attention, memory, vigilance, reaction time and behavior, MHE is detected using sensitive neuropsychometric and neurophysiological tests [6]. As much as $80 \%$ of patients with end-stage liver disease are affected by MHE, which severely impacts on the patients' capability to drive a car, to continue working, and their ability to function daily, overall affecting their health-related quality of life [7].

Brain edema is an accumulation of water within the cerebral tissue (intracellular and/or extracellular). It occurs as a result of an increase in osmolarity and/or compromised volume regulatory responses. Impairment in the efflux of brain organic osmolytes, such as polyols (myo-inositol) and amino acids (taurine, glutamate, glutamine), fails to compensate for the increased osmolarity and, therefore, an increase in brain water content prevails [8].

Ammonia is considered a major pathogenic factor in the development of HE [9]. The brain solely relies on the amidation of glutamate catalyzed by the enzyme glutamine synthetase (GS) to detoxify ammonia. However, because the ailing liver is incapable of efficiently clearing it, the increase in blood ammonia leads to neurotoxic levels of ammonia. Therefore, during hyperammonemia, elevated brain ammonia leads to an increase in brain glutamine [10], a pathway believed to be involved in the development of brain edema and HE.

Lactate is another pathogenic factor demonstrated to be implicated in HE [11]. Lactate is a product of anaerobic glycolysis, but also a metabolite used by neurons as an energetic substrate [12]. An increase in cerebral lactate, due to increased glycolytic activity and/or energy failure, can osmotically induce an increase in water influx in the brain, and thus lead to brain edema, as demonstrated in numerous neuropathies, including cerebral ischemia [13]. However, the role of lactate in the pathogenesis of brain edema in HE due to CLD remains undetermined.

The present study aims to explore the pathophysiological mechanisms implicated in brain edema in cirrhotic rats with MHE, with an emphasis on the role of lactate and glutamine and brain osmolytes, including myo-inositol and taurine. The 6-week bile-duct ligated (BDL) rat is a well-characterized animal model of liver fibrosis and necrosis [14], which develops both brain edema and MHE [15] and [16]. In this model, we investigated the metabolic fluxes of ${ }^{13} \mathrm{C}$-labelled glucose, and focused on de novo synthesis of lactate and glutamine using nuclear magnetic resonance (NMR) spectroscopy, an excellent technique to quantify cellular metabolic fluxes ( Fig. 1A). As ${ }^{13} \mathrm{C}$ represents only $1.1 \%$ of natural carbon, sample enrichment following injection of ${ }^{13} \mathrm{C}$-labelled glucose permits the evaluation of glucose metabolic fluxes through the glycolytic pathway and the tricarboxylic acid (TCA) cycle [17]. In order to evaluate the interplay between lactate, glutamine and organic osmolytes, BDL rats were treated with AST-120 (oral ammonia adsorbent engineered activated carbon microspheres) and dichloroacetate (DCA), a lactate synthesis inhibitor.

Bosoi, C.R. et al., 2014. Increased brain lactate is central to the development of brain edema in rats with chronic liver disease. Journal of Hepatology, 60(3), p.554-560. 


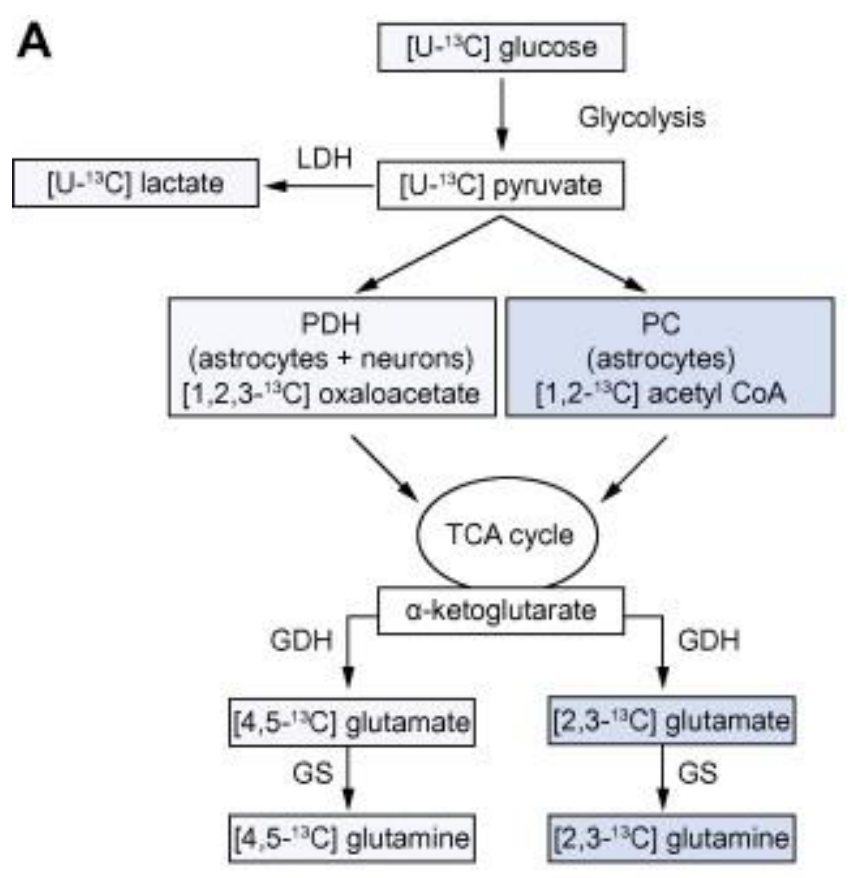

B

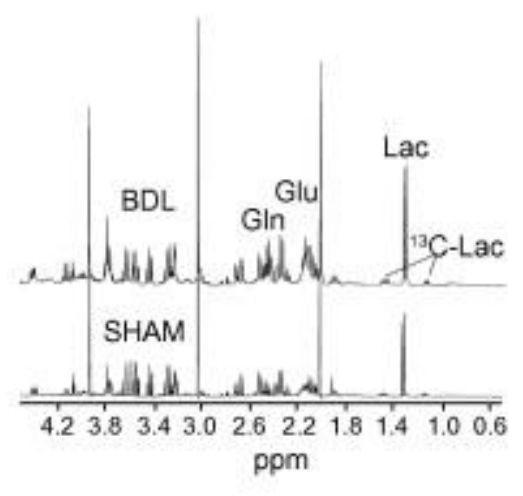

C

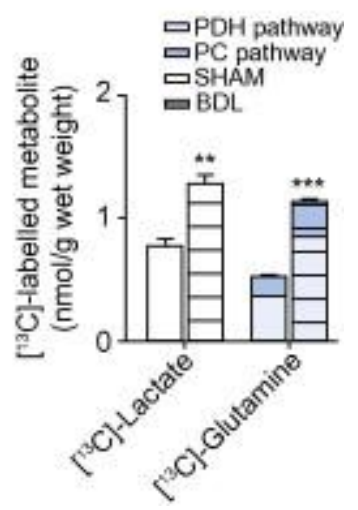

Fig. 1. ${ }^{13} \mathrm{C}$ nuclear magnetic resonance spectroscopy. (A) Schematic representation of ${ }^{13} \mathrm{C}$-labelled metabolites formation from $\left[\mathrm{U}_{-}{ }^{13} \mathrm{C}\right]$-glucose. Glucose is metabolized via glycolysis into pyruvate which either produces lactate through lactate dehydrogenase (LDH) or enters the TCA cycle. Upon entering the TCA cycle, pyruvate is metabolized via pyruvate dehydrogenase (PDH), found in both astrocytes and neurons, or via pyruvate carboxylase (PC), found exclusively in the astrocytes. The $\left[\mathrm{U}_{-}{ }^{13} \mathrm{C}\right]$-pyruvate formed from $\left[\mathrm{U}_{-}{ }^{13} \mathrm{C}\right]$-glucose through $\mathrm{PDH}$ results in $\left[1,2-{ }^{13} \mathrm{C}\right]$-acetyl-CoA, and further via $\alpha$-ketoglutarate to $\left[4,5-{ }^{13} \mathrm{C}\right]$ labelled glutamate and glutamine; through PC it results in $\left[1,2,3-{ }^{13} \mathrm{C}\right]$-oxaloacetate and $\left[2,3-{ }^{13} \mathrm{C}\right]$ labelled metabolites. Glutamate is produced from the TCA intermediate $\alpha$-ketoglutarate via $\alpha$-ketoglutarate dehydrogenase and via amidation by glutamine synthetase (GS) can form glutamine. LDH, lactate dehydrogenase; PDH, pyruvate dehydrogenase; PC, pyruvate carboxylase; TCA cycle, tricarboxylic acid cycle; GS, glutamine synthetase. (B) Representative ${ }^{13} \mathrm{C}$ nuclear magnetic resonance spectra in rats with bile-duct ligation (BDL) compared to SHAM-operated controls. (C) Cerebral de novo synthesis from ${ }^{13} \mathrm{C}$-glucose of lactate and glutamine in rats with bile-duct ligation (BDL) compared to SHAM-operated controls. Light blue: $\left[4,5-{ }^{13} \mathrm{C}\right]$-glutamine formed through PDH; dark blue: $\left[2,3-{ }^{13} \mathrm{C}\right]-$ glutamine formed through PC. Data are expressed as mean \pm SEM. ${ }^{* *} p<0.01,{ }^{* * *} p<0.001$, significantly different from SHAM.

Bosoi, C.R. et al., 2014. Increased brain lactate is central to the development of brain edema in rats with chronic liver disease. Journal of Hepatology, 60(3), p.554-560. 


\section{MATERIALS AND METHODS}

\section{Animal models}

Male Sprague-Dawley rats (250-275 g) (Charles River, St-Constant, QC) were randomly selected to either bile-duct ligation (BDL) or SHAM operation, and studied 6 weeks following surgery [15]; for cerebral ammonia measurement, cerebrospinal fluid was collected from the cisterna magna, as previously described [18]. All the experiments were performed following the Guidelines of the Canadian Council on Animal Care, and were approved by the Animal Protection Committee of the CRCHUM.

\section{Nuclear magnetic resonance Administration of $\left[\mathrm{U}^{13}{ }^{13} \mathrm{C}\right]$-glucose}

6 weeks after surgery BDL and SHAM rats received $\left[\mathrm{U}^{13} \mathrm{C}\right]$-glucose $(500 \mathrm{mg} / \mathrm{kg}$, intraperitoneally; Cambridge Isotope Laboratories, Andover, MA) and were sacrificed exactly 30 minutes later by decapitation. Arterial blood glucose levels were monitored 3 days before sacrifice; however, no glycaemia differences were found between SHAM and BDL operated rats. Therefore, there was no need to correct glucose levels. [U- ${ }^{13} \mathrm{C}$ ]-glucose is transformed through glycolysis in [U- $\left.{ }^{13} \mathrm{C}\right]-$ pyruvate. This can either enter the TCA cycle or form $\left[\mathrm{U}-{ }^{13} \mathrm{C}\right]$-lactate through lactate dehydrogenase. Upon entering the TCA cycle, glucose-derived pyruvate is metabolized either by pyruvate dehydrogenase (PDH), the key enzyme for mitochondrial energy found in both astrocytes and neurons, or by pyruvate carboxylase (PC), an important anaplerotic enzyme that replenishes TCA cycle intermediates, found exclusively in astrocytes. The $\left[\mathrm{U}-{ }^{13} \mathrm{C}\right]$-pyruvate formed from $[\mathrm{U}-$ $\left.{ }^{13} \mathrm{C}\right]$-glucose metabolised through PDH results in $\left[1,2-{ }^{13} \mathrm{C}\right]$-acetyl-CoA, and further via $\alpha$-ketoglutarate to $\left[4,5-{ }^{13} \mathrm{C}\right]$-labelled glutamate and glutamine, whereas $\left[\mathrm{U}-{ }^{13} \mathrm{C}\right]$-pyruvate metabolised through $\mathrm{PC}$ results in $\left[1,2,3-{ }^{13} \mathrm{C}\right]$-oxaloacetate and $[2,3-$ ${ }^{13} \mathrm{C}$-labelled metabolites. These reactions allowed for the quantification of fluxes through these pathways [17] (Fig. 1A).

\section{NMR spectroscopy [19]}

Immediately after sacrifice, the brains were snap frozen in liquid nitrogen and stored at $-80{ }^{\circ} \mathrm{C}$ until measurements were performed. Water-soluble metabolites were extracted with $7 \%$ perchloric acid. The lyophilized water-soluble samples were dissolved in $\mathrm{D}_{2} \mathrm{O}$, centrifuged, and adjusted to $\mathrm{pH}$ 7.2. NMR spectra were recorded on a DRX-600 Bruker spectrometer. ${ }^{1} \mathrm{H}-$ NMR spectra were recorded with a 5-mm H,C,N-inverse-triple-resonance probe, flip angle $40^{\circ}$, repetition time $15 \mathrm{~s}$, spectral width $7183 \mathrm{~Hz} .{ }^{13} \mathrm{C}-\mathrm{NMR}$ spectra were recorded with a $5 \mathrm{~mm}{ }^{1} \mathrm{H} /{ }^{13} \mathrm{C}$ dual probe, repetition time $2.5 \mathrm{~s}$, flip angle $27^{\circ}$, composite pulse decoupling with WALTZ-16, spectral width 47,619 Hz. Total brain glutamine, glutamate, myo-inositol and taurine were determined on ${ }^{1} \mathrm{H}-\mathrm{NMR}$ spectra, while ${ }^{13} \mathrm{C}$-labelled lactate and glutamine were determined on ${ }^{13} \mathrm{C}-\mathrm{NMR}$ spectra.

\section{Therapeutic interventions}

\section{Ast-120}

AST-120 (Ocera Therapeutics, San Diego, CA), engineered carbon microspheres, were administered by gavage $(1 \mathrm{~g} / \mathrm{kg} / \mathrm{d}$; concentration $1 \mathrm{~g} / 10 \mathrm{ml}$ of $2 \%$ methylcellulose) every 12 hours, for a period of 6 weeks, beginning day 1 after surgery in both SHAM and BDL rats [15]. As controls, another group of SHAM and BDL rats were treated with equivalent volumes of methylcellulose.

\section{Dichloroacetate}

Dichloroacetate (DCA; Sigma-Aldrich), a lactate synthesis inhibitor, was administered by intraperitoneal injection at a dose of $25 \mathrm{mg} / \mathrm{kg} / \mathrm{d}$ (concentration of $25 \mathrm{mg} / \mathrm{ml}$ saline) for 7 days (starting day 35 after surgery) in both SHAM and BDL rats. This PDH kinase inhibitor results in a dephosphorylation of PDH and hence increases its activity. As a result, the flux of pyruvate into the TCA cycle increases, consequently decreasing lactate synthesis by shifting lactate dehydrogenase activity from lactate to pyruvate production [20].

At 6 weeks, brain tissue was collected to measure lactate, glutamine and brain edema as described below.

Bosoi, C.R. et al., 2014. Increased brain lactate is central to the development of brain edema in rats with chronic liver disease. Journal of Hepatology, 60(3), p.554-560. 


\section{Ammonia measurement}

Ammonia was assessed in cerebrospinal fluid using a commercial kit (Sigma-Aldrich). The kit is based on the reaction of ammonia with $\alpha$-ketoglutarate and reduced nicotinamide adenine dinucleotide phosphate in the presence of l-glutamate dehydrogenase. Oxidation rate of reduced nicotinamide adenine dinucleotide phosphate was recorded by the absorbance decrease at $340 \mathrm{~nm}$. Ammonia concentration was calculated according to the manufacturer's protocol.

\section{Lactate measurement}

The frontal cortex was dissected and homogenized in lysis buffer (50 mM Tris pH 7.5, $1 \mathrm{mM}$ EDTA, 1/500 cold Protease Inhibitor Cocktail (Roche, Indianapolis, IN)). Lactate levels were assessed following its oxidation by lactate oxidase to pyruvate and hydrogen peroxide, which reacts with AmplexRed (10-acetyl-3,7-dihydroxyphenoxazine) and releases resorufin, a fluorescent oxidation product. Fluorescence was read at $530 \mathrm{~nm}$ excitation and $590 \mathrm{~nm}$ emission wavelengths, and lactate levels were calculated based on a standard curve of known lactate concentrations.

\section{Glutamine measurement}

Brain frontal cortex samples were analyzed using the Agilent 1100 Chemstation reverse-phase HPLC system (Agilent Technologies, Germany) with fluorescence detection as previously described [21]. Glutamine concentration was calculated by peak area analysis with an automated integrator (Agilent Technologies, Germany), based on standard curves and internal standards.

\section{Brain water content}

Frontal cortex brain water content was measured using the sensitive densitometry technique described by Marmarou et al., as previously reported by our group [15] and [22].

\section{Statistical analysis}

Data are expressed as mean \pm standard error of the mean (SEM). Significance of difference was tested with Student's $t$ test or ANOVA, followed by Newman-Keuls post-test; correlation was calculated with Spearman test using GraphPad Prism4 (La Jolla, CA). Probability values $p<0.05$ were considered to be statistically significant.

\section{RESULTS}

\section{Cerebral lactate and glutamine in BDL rats}

Six week BDL rats demonstrated a significant increase in brain lactate $(254.6 \pm 11.1 \mu \mathrm{M} / 100 \mu \mathrm{g}$ protein $v s$. SHAM: $111.7 \pm 7.1 \mu \mathrm{M} / 100 \mu \mathrm{g}$ protein, $p<0.001)$, and brain glutamine $(8.42 \pm 12.77 \mu \mathrm{mol} / \mathrm{g}$ tissue $v s$. SHAM: $4.42 \pm 3.38 \mu \mathrm{mol} / \mathrm{g}$ tissue, $p<0.01)$.

\section{De novo synthesis of cerebral lactate and glutamine from ${ }^{13} \mathrm{C}$-labelled glucose in $\mathrm{BDL}$ rats} Following ${ }^{13} \mathrm{C}$-labelled glucose administration, de novo synthesis of lactate and glutamine significantly increased 1.6- and 2.2-fold in BDL vs. SHAM-operated control rats ( Fig. 1B and C).

Determination of the position of the ${ }^{13} \mathrm{C}$-labelled carbon in de novo synthetized glutamine showed that the flux through PDH (2.3-fold increase) and PC (1.8-fold increase) was higher in BDL vs. SHAM rats ( Fig. 1C).

\section{Brain osmolytes in BDL rats}

${ }^{1} \mathrm{H}$ is naturally present in tissues and through its detection, numerous molecules can thus be simultaneously quantified by ${ }^{1} \mathrm{H}$ NMR spectroscopy. The cerebral osmolyte pool, obtained by adding all measured osmolytes, was 1.3 -fold higher in BDL $v s$. SHAM-operated control rats ( Table 1). In BDL rats, a significant increase in brain glutamine $(128 \% ; p<0.01 v s$. SHAM) and glutamate ( $26 \% ; p<0.01 v s$. SHAM) was accompanied by a significant decrease in myo-inositol (23\%; $p<0.001$ vs. SHAM). Brain taurine remained unchanged.

Bosoi, C.R. et al., 2014. Increased brain lactate is central to the development of brain edema in rats with chronic liver disease. Journal of Hepatology, 60(3), p.554-560. 
Table 1. ${ }^{1} \mathrm{H}$-NMR concentration of brain osmolytes in rats with bile-duct ligation (BDL) compared to respective SHAM-operated controls.

\begin{tabular}{lll}
\hline & BDL-SHAM & BDL \\
\hline Glutamine ( $\mu \mathrm{mol} / \mathrm{g}$ wet weight) & $4.67 \pm 0.28$ & $10.67 \pm 0.98^{* *}$ \\
Glutamate ( $\mu \mathrm{mol} / \mathrm{g}$ wet weight) & $11.55 \pm 0.48$ & $14.56 \pm 0.99^{* *}$ \\
Myo-inositol ( $\mu \mathrm{mol} / \mathrm{g}$ wet weight) & $4.31 \pm 0.14$ & $3.34 \pm 0.14^{* * *}$ \\
Taurine ( $\mu \mathrm{mol} / \mathrm{g}$ wet weight) & $4.71 \pm 0.43$ & $5.48 \pm 1.73$ \\
Total & $25.20 \pm 1.33$ & $34.00 \pm 2.95$ \\
\hline
\end{tabular}

Data are expressed as mean \pm SEM. ${ }^{* *} p<0.01,{ }^{* * *} p<0.001$, significantly different from SHAM.

\section{Effect on cerebral lactate and glutamine following ammonia reduction}

Administration of AST-120, engineered activated carbon microspheres with a high nonspecific adsorptive surface area acting within the gut, lead to a significant decrease in ammonia in the brain (cerebrospinal fluid) (Fig. 2A) and to an attenuation in brain edema (Fig. 2B). Moreover, AST-120-treated BDL rats resulted in a significant reduction in brain lactate $v s$. non-treated BDL rats. However, lactate remained significantly high in AST-120 treated BDL rats compared to SHAM-operated controls $(p<0.001)$ ( Fig. 2 C). Contrary to lactate, the lowering of ammonia by AST-120 did not reduce brain glutamine levels ( Fig. 2D).

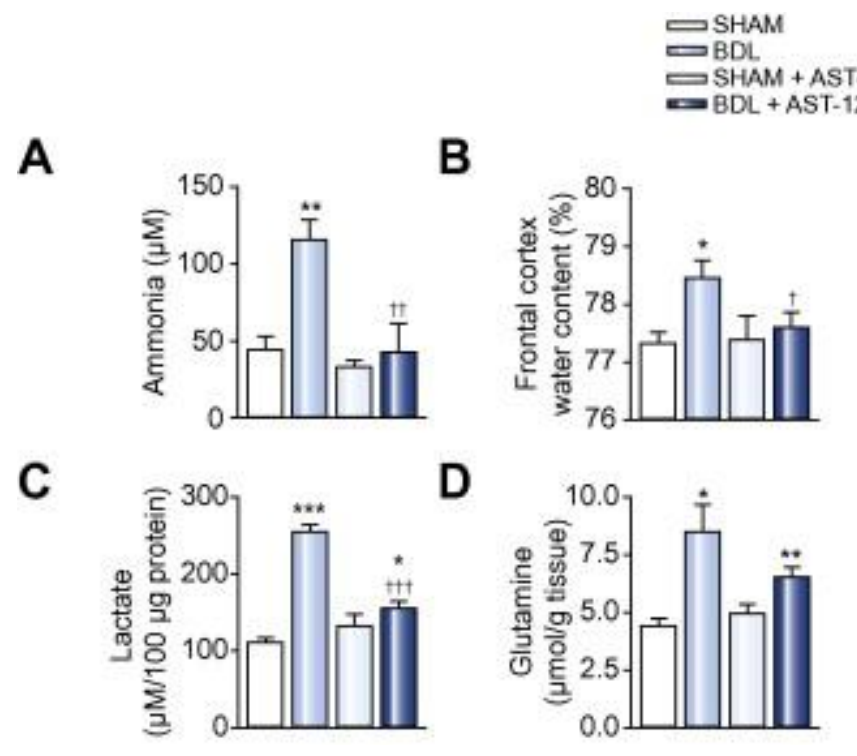

Fig. 2. Effect of AST-120 (spherical carbon adsorbent). (A) cerebrospinal fluid ammonia; (B) frontal cortex brain water content; (C) frontal cortex lactate and (D) frontal cortex glutamine levels in bile-duct ligation (BDL) rats compared to treated and non-treated SHAM-operated controls and non-treated BDL rats. Data are expressed as mean \pm SEM. ${ }^{*} p<0.05$, ${ }^{* *} p<0.01,{ }^{* * *} p<0.001$, significantly different from SHAM; ${ }^{\dagger} p<0.05,{ }^{\dagger \dagger} p<0.01,{ }^{\dagger \dagger} p<0.001$, significantly different from non-treated BDL.

\section{Effect on brain edema following lactate reduction}

In order to identify the precise role of lactate in the pathogenesis of brain edema, BDL rats were treated with DCA, a lactate synthesis inhibitor. The DCA treatment reduced brain water content in BDL rats $(p<0.05)$ ( Fig. 3A) and normalized brain lactate levels ( Fig. 3B). Cerebral glutamine decreased following DCA treatment to levels that were not significantly different vs. either non-treated BDL rats or SHAM-operated controls ( Fig. 3C). To verify if these modifications are not due to a direct effect of DCA on ammonia levels, those were assessed and were found to be similar in non-treated and DCA-

Bosoi, C.R. et al., 2014. Increased brain lactate is central to the development of brain edema in rats with chronic liver disease. Journal of Hepatology, 60(3), p.554-560. 
treated BDL rats ( Fig. 3D). In addition, DCA treatment did not have a beneficial effect on the liver function as no change in liver enzymes (aspartate aminotransferase (AST) and alanine aminotransferase (ALT)) was found between non-treated and DCA-treated BDL rats (AST: DCA-treated: 301.3 \pm 141.7 U/L vs. non-treated:364.4 \pm 64.7 U/L, $p$ >0.05; ALT: DCAtreated: $65.2 \pm 11.8 \mathrm{U} / \mathrm{L} v s$. non-treated:73.4 $\pm 7.1 \mathrm{U} / \mathrm{L}, p>0.05)$.

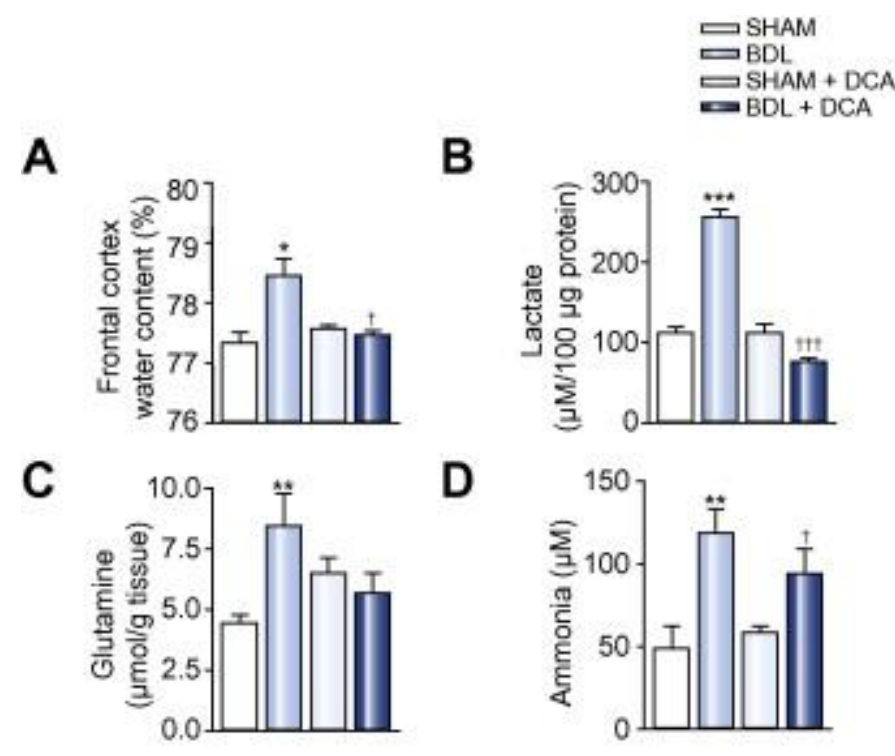

Fig. 3. Effect of dichloroacetate (DCA) (lactate synthesis inhibitor). (A) frontal cortex water content; (B) frontal cortex lactate; (C) frontal cortex glutamine and (D) cerebrospinal fluid ammonia levels in bile-duct ligation (BDL) rats compared to treated and non-treated SHAM-operated controls and non-treated BDL rats. Data are expressed as mean \pm SEM. ${ }^{*} p<0.05$, ${ }^{* *} p<0.01,{ }^{* * *} p<0.001$, significantly different from SHAM; ${ }^{\dagger} p<0.05,{ }^{\dagger \dagger} p<0.001$, significantly different from non-treated BDL.

\section{Cerebral lactate temporal resolution}

To thoroughly understand the relationship between ammonia, lactate and glutamine, we monitored the changes of these three pathogenic factors at weeks 2, 4, and 6, and characterized their temporal resolution in relation to the appearance of brain edema. No differences were found between SHAM-operated controls sacrificed at 2, 4, and 6 weeks. After 2 weeks of BDL, neither brain lactate, brain ammonia nor brain water content was significantly elevated. However, brain glutamine levels were significantly higher compared to SHAM-operated controls. After 4 weeks of BDL, along with no evidence of brain edema, a significant increase in brain lactate and ammonia was observed ( $v s$. 2 weeks), with a similar increase in the levels of glutamine as at 2 weeks. Six weeks following BDL, brain edema appeared, along with a significant further increase in brain lactate and ammonia, compared to 4 weeks. No further increase in glutamine levels was demonstrated in comparison to weeks 2 and 4 ( Fig. 4A). Using the data obtained at 2, 4, and 6 weeks after BDL and SHAM-operated controls, a significant correlation was calculated between cerebrospinal fluid ammonia and lactate $(\mathrm{r}=0.5447, p<0.05$; Fig. 4B). Ammonia levels did not significantly correlate with cerebral glutamine ( $r=-0.1255$, Fig. 4C).

Bosoi, C.R. et al., 2014. Increased brain lactate is central to the development of brain edema in rats with chronic liver disease. Journal of Hepatology, 60(3), p.554-560. 


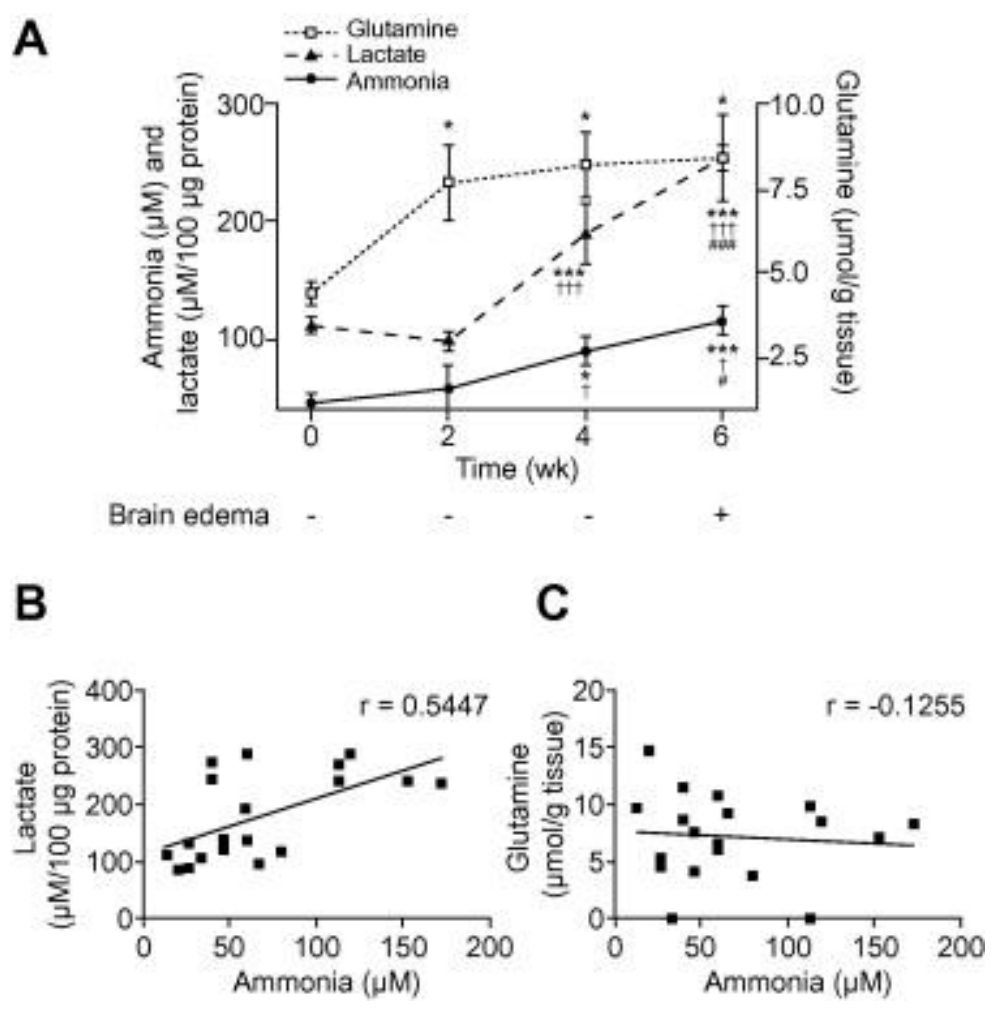

Fig. 4. Correlations between lactate, glutamine, ammonia and brain edema. (A) Temporal resolution of lactate, glutamine, ammonia and brain edema over 6 weeks in rats with bile-duct ligation (BDL) compared to respective SHAMoperated controls. The time point 0 represents the value for SHAM-operated controls sacrificed 6 weeks after surgery. (B) Correlation between changes in cerebrospinal fluid ammonia and brain lactate in BDL rats following 2, 4, and 6 weeks after the intervention. (C) Correlation between changes in cerebrospinal ammonia and cerebral glutamine in BDL rats following 2,4 , and 6 weeks after the intervention. Data are expressed as mean \pm SEM. ${ }^{*} p<0.05,{ }^{* * *} p<0.001$, significantly different from SHAM; ${ }^{\dagger} p<0.05,{ }^{\dagger \dagger} p<0.001$, significantly different from BDL 2 weeks; ${ }^{\#} p<0.05$, ${ }^{\# \# \# ~} p<0.001$, significantly different from BDL 4 weeks.

\section{DISCUSSION}

Results of the present study reveal for the first time in the setting of CLD that increased cerebral lactate, and not increased glutamine, is a key factor in the pathogenesis of brain edema. NMR spectroscopy revealed an increase in both lactate and glutamine de novo synthesis in the brain from ${ }^{13} \mathrm{C}$-glucose in cirrhotic rats with brain edema and MHE. The importance of lactate in the development of brain edema was established following the treatment with AST-120. These orally administered carbon microspheres, in addition to attenuating hyperammonemia and normalizing brain water content in BDL rats, also decreased lactate levels in the brain. To confirm the crucial role of lactate, following the reduction of brain lactate levels in BDL rats treated with DCA (lactate synthesis inhibitor), the cerebral content of water was attenuated. Furthermore, following the same treatment regimens, no change in brain glutamine levels was found, suggesting glutamine does not contribute to an increase in cerebral water in cirrhotic rats. Taken together, these findings underscore the importance of lactate over glutamine in the development of brain edema in CLD.

There is substantial evidence that links increased cerebral lactate to severe HE. In patients with fulminant hepatic failure, it has been shown that increases in extracellular lactate correlate with rises in intracranial pressure [23]. These findings have been supported in rats with acute liver failure, where by using ${ }^{13} \mathrm{C}$-NMR spectroscopy, it was found that an increased $d e$ novo synthesis of lactate from glucose correlated with severe HE [24] and [25], and that the progression from pre-coma to

Bosoi, C.R. et al., 2014. Increased brain lactate is central to the development of brain edema in rats with chronic liver disease. Journal of Hepatology, 60(3), p.554-560. 
coma stage is associated with the development of brain edema and a marked increase in cerebral lactate [26]. Moreover in acute liver failure, therapeutic interventions, such as mild hypothermia and albumin dialysis, have shown to reduce cerebral lactate along with brain edema and the development of severe HE (coma and intracranial hypertension) [24], [27] and [28]. Furthermore, in the setting of CLD, a 1.37-fold increase in lactate in the cerebrospinal fluid has been found in patients with end-stage liver disease and overt/severe HE (grades 3 and 4) [29]. Chronic hyperammonemic rats (induced following 4week portacaval anastomosis) injected with a toxic dose of ammonia precipitates severe HE (coma), which is accompanied with brain edema and an increase in brain lactate [30] and [31]. However, notwithstanding concrete evidence associating elevated concentrations of brain lactate and severe HE (intracranial hypertension, coma), the role of lactate in the pathogenesis of MHE remains elusive.

MHE is a clinically important entity that affects up to $80 \%$ of patients with end-stage liver disease, placing them at a 4times higher risk of developing overt HE [32]. For this, our study describes, for the first time, the implications of lactate in the pathogenesis of brain edema and cirrhosis-induced MHE. Our results demonstrate that increased cerebral lactate due to de novo synthesis from glucose plays a vital role in the development of brain edema in cirrhotic rats. Interestingly, the overall increase in ${ }^{13} \mathrm{C}$-labelled de novo synthesis of lactate in BDL rats with brain edema and MHE is 1.7-fold, compared to the 4.0-fold increase observed in acute liver failure rats with brain edema and severe HE (coma) [25]. Moreover, brain edema is attenuated following DCA treatment.

These results, together with previous data arising from rats with acute liver failure, may suggest that brain lactate not only plays a significant role in the development of brain edema, but might also correlate with the severity of HE. It has been proposed in the setting of CLD, where intracranial hypertension is rarely observed, that the degree of brain edema is of "low-grade" [2]. This implies a strong relationship between lactate levels, degree of brain edema and severity of HE.

Liver failure leads to a significant reduction in the capacity to detoxify ammonia and, as a result, the developing hyperammonemia causes a rise in brain ammonia levels. Ammonia toxicity has been demonstrated to lead to an increase in lactate by inhibiting enzyme $\alpha$-ketoglutarate dehydrogenase in the TCA cycle [33]. This in turn stimulates glutamate dehydrogenase, an alternative pathway to remove ammonia through the amidation of $\alpha$-ketoglutarate to glutamate and subsequently to glutamine. Our results demonstrate an increase in glucose-derived glutamine in BDL rats, supporting stimulation of this pathway. In addition, we observed an increase in glucose-derived lactate, and an increase in glycolysis flux, possibly a result of ammonia-stimulated phosphofructokinase activity [34]. However, in spite of these TCA cycle alterations (inhibition of $\alpha$-ketoglutarate dehydrogenase), ATP levels remain maintained [35] and [36]. Therefore, ammoniainduced increase in brain lactate is a not a result of energy failure (activated anaerobic metabolism); rather, the increase in lactate synthesis may occur as a compensatory mechanism to maintain ATP levels.

It is well documented that the shuttling of lactate between astrocytes and neurons plays an important role in brain physiology. Astrocyte-derived lactate is used by surrounding neurons as an energy substrate, coupling cerebral glucose metabolism to neuronal activity [12]. Hence, dysregulation of the astrocyte-neuron lactate shuttle due to changes in lactate metabolism results in altered lactate homeostasis and leads to brain edema and cerebral dysfunction. Indeed, affecting lactate homeostasis in the brain can lead to differential lactate compartmentalization and changes in osmolarity. It has previously been shown that exposure of astrocytes (cell type shown to selectively exhibit swelling in HE) to pathophysiologically relevant concentrations of lactate can lead to significant swelling [37]. Furthermore, increased lactate production not only leads to osmotic stress, but also generates more water per ATP formed than oxidative phosphorylation [38]. This supports our results that, in the brains of BDL rats, an increase in lactate synthesis is a pivotal factor in the development of brain edema.

In an effort to remove ammonia, the brain depends on the enzyme glutamine synthetase (GS), which is specifically found in astrocytes [39]. Using NMR and administering ${ }^{13} \mathrm{C}$-labelled glucose, the de novo synthesis of metabolites from glucose can be quantified by evaluating the position of ${ }^{13} \mathrm{C}$. This helps distinguish if glutamine is synthetized from ${ }^{13} \mathrm{C}$-labelled glucose via PDH (oxidative pathway found in both neurons and astrocytes) or via PC (anaplerotic pathway found exclusively in astrocytes) [17]. In BDL rats, an increase in de novo synthesis of glutamine via both PDH and PC was demonstrated, which

Bosoi, C.R. et al., 2014. Increased brain lactate is central to the development of brain edema in rats with chronic liver disease. Journal of Hepatology, 60(3), p.554-560. 
suggests that both oxidative and anaplerotic pathways were upregulated. This provides evidence that neuronal glucosederived glutamate is synaptically released (excitatory neurotransmission), and is subsequently captured by astrocytes and expended to detoxify ammonia through GS.

During conditions of hyperammonemia, intracellular glutamine trapping (accumulation) is believed to contribute to astrocyte hypertonicity, astrocyte swelling and brain edema [40]. It has been previously shown that exposing primary cultured astrocytes to ammonia results in cell swelling, an outcome that is abolished following the inhibition of GS with methionine-sulfoximine (MSO) [41]. Moreover, in ammonia-infused portacaval shunted rats, pre-treatment with MSO leads to an attenuation of cerebral glutamine levels and ameliorated brain edema [42]. However, in addition to its osmotic influence, glutamine is believed to be toxic and has demonstrated to impair mitochondrial function through opening of the mitochondrial transition pore, thus causing astrocyte swelling [43]. In the present study, we found glutamine levels to be persistently high following brain edema resolution with treatments AST-120 and DCA. This suggests glutamine accumulation is not an important pathogenetic factor in the development of brain edema in HE. Interestingly, this observation has also been described in animal models of acute liver failure, in which high cerebral glutamine levels persisted following ammonia-lowering treatments and resolution of ICP, brain edema and HE [19] and [24]. Moreover, 4week hyperammonemic portacaval-shunted rats, with an increase in brain glutamine, do not develop brain edema [18]. Therefore, taken together, there is strong, accumulating evidence dictating that increased cerebral glutamine does not play a vital role in the development of brain edema in liver failure.

Myo-inositol and taurine, considered to be major organic osmolytes, play an important role in cell volume regulation. They are released into the extracellular space, compensating for intracellular hypertonicity, thus preventing cell swelling and the development of brain edema [44]. In hyperammonemic portacaval shunted rats, it is stated that the reason brain edema does not develop, even in the presence of increased brain glutamine, is a result of a substantial compensatory decrease in myoinositol, taurine and glutamate [45]. Interestingly, also in portacaval shunted rats, lack of brain edema is accompanied with no increase in brain lactate [31]. In the present study, 6-week BDL rats developed brain edema, along with increases in both brain glutamine and lactate. A decrease in brain myo-inositol, but no significant change in taurine, was observed. As a result, higher osmolarity was calculated in BDL rats compared to SHAM-operated controls. This suggests that impaired brain osmoregulation, possibly the result of exhaustive release of osmoregulators, cannot compensate for the increase in both brain glutamine and lactate. This osmolyte profile has been similarly observed in rats with acute liver failure, where the sum of all brain osmolytes (including glutamine) exceeded the decrease in myo-inositol and taurine at coma stage (in the presence of brain edema) [19].

To further understand the relation and interplay between lactate, glutamine and ammonia in the development of brain edema, we studied the temporal resolution of these factors in 6-week cirrhotic rats. Two weeks following BDL, brain edema was not present. A surge in cerebral glutamine was observed with no significant elevation in ammonia or lactate. At 4 weeks, with brain edema still not present, a significant increase in ammonia and lactate was detected with no further increase in glutamine observed. At 6 weeks, the appearance of brain edema was associated with an additional rise in ammonia and lactate levels, but no further rise in glutamine levels. Taken all together, a significant correlation was found between brain ammonia and lactate, but not between brain ammonia and glutamine. The sudden rise in glutamine at 2 weeks, followed by no significant additional increase at weeks 4 and 6 may be the result of saturation or inhibition of GS activity [46], [47], [48] and [49]. A reduction in GS activity is not due to glutamate being a limiting factor, since increases in brain glutamate were found in BDL $v s$. SHAM-operated rats ( Table 1). Our results sustain that increased brain lactate (and not brain glutamine) is a consequence of hyperammonemia-induced increased brain ammonia, which leads to brain edema. In the context of HE, increased glutamine levels are commonly observed even when brain edema is not present, as observed in portacaval-shunted rats [45]. However, in other neurological diseases such as cerebral ischemia, increased brain lactate levels have been associated with the development of brain edema, while brain glutamine levels were decreased [50], [51] and [52]. Although our study was performed in frontal cortex, it is possible other cerebral regions may be affected dissimilarly. For example, it was demonstrated in cerebellum of rats with acute liver failure that brain edema precedes an

Bosoi, C.R. et al., 2014. Increased brain lactate is central to the development of brain edema in rats with chronic liver disease. Journal of Hepatology, 60(3), p.554-560. 
increase in lactate [53]. Different regions within the brain in relation to lactate levels and water content remain to be investigated.

In conclusion, the present study demonstrates for the first time that an increase in brain lactate, and not brain glutamine, is a pivotal factor involved in the pathogenesis of brain edema in end-stage liver disease. In addition, the results of the present study also suggest that impaired compensatory osmoregulatory mechanisms may be a contributing factor in the development of brain edema in CLD. Currently, lowering ammonia levels represents the primary treatment strategy in patients with HE and CLD [54]. The present results demonstrate AST-120 is an efficient ammonia-lowering strategy as has been previously demonstrated [15]. However, in addition, the results of the present study also reveal DCA as a potential treatment of HE. This lactate synthesis inhibitor has previously demonstrated a long-term safety profile in patients with congenital lactic acidosis [55] and beneficial effects with no adverse reactions in other diseases such as cancer [56] and chronic obstructive pulmonary disease [57]. Therefore, DCA can rapidly provide a promising therapeutic approach for the management of patients with end-stage liver disease.

\section{Conflict of interest}

The authors who have taken part in this study declared that they do not have anything to disclose regarding funding or conflict of interest with respect to this manuscript.

\section{Acknowledgments}

This work was supported by a grant from the Canadian Institutes of Health Research. CRB is a recipient of a Doctoral research award from the Fonds de recherche du Québec - Santé. The authors thank Ocera Therapeutics, San Diego, CA for providing AST-120.

\section{REFERENCES}

[1] Clemmesen JO, Larsen FS, Kondrup J, Hansen BA, Ott P. Cerebral herniation in patients with acute liver failure is correlated with arterial ammonia concentration. Hepatology. 1999;29:648-653.

[2] Häussinger D. Low grade cerebral edema and the pathogenesis of hepatic encephalopathy in cirrhosis. Hepatology. 2006;43:1187-1190.

[3] Shah NJ, Neeb H, Kircheis G, Engels P, Häussinger D, Zilles K. Quantitative cerebral water content mapping in hepatic encephalopathy. Neuroimage. 2008;41:706-717.

[4] Lodi R, Tonon C, Stracciari A, Weiger M, Camaggi V, Iotti S, et al. Diffusion MRI shows increased water apparent diffusion coefficient in the brains of cirrhotics. Neurology. 2004;62:762-766.

[5] Sugimoto R, Iwasa M, Maeda M, Urawa N, Tanaka H, Fujita N, et al. Value of the apparent diffusion coefficient for quantification of low-grade hepatic encephalopathy. Am. J. Gastroenterol. 2008;103:1413-1420.

[6] Córdoba J. New assessment of hepatic encephalopathy. J. Hepatol. 2011;54:1030-1040.

[7] Stewart CA, Smith GE. Minimal hepatic encephalopathy. Nat Clin Pract Gastroenterol Hepatol. 2007;4:677-685.

[8] McManus ML, Churchwell KB, Strange K. Regulation of Cell Volume in Health and Disease. New England Journal of Medicine. 1995;333:1260-1267.

[9] Felipo V, Butterworth RF. Neurobiology of ammonia. Prog. Neurobiol. 2002;67:259-279.

[10] Pasantes-Morales H, Cruz-Rangel S. Brain volume regulation: osmolytes and aquaporin perspectives. Neuroscience. 2010;168:871-884.

[11] Rose CF. Increase brain lactate in hepatic encephalopathy: cause or consequence? Neurochem. Int. 2010;57:389-394.

[12] Pellerin L, Magistretti PJ. Sweet sixteen for ANLS. J. Cereb. Blood Flow Metab. 2012;32:1152-1166.

[13] Helbok R, Ko S-B, Schmidt JM, Kurtz P, Fernandez L, Choi HA, et al. Global cerebral edema and brain metabolism after subarachnoid hemorrhage. Stroke. 2011;42:1534-1539.

[14] Bataller R, Gäbele E, Parsons CJ, Morris T, Yang L, Schoonhoven R, et al. Systemic infusion of angiotensin II exacerbates liver fibrosis in bile duct-ligated rats. Hepatology. 2005;41:1046-1055.

[15] Bosoi CR, Parent-Robitaille C, Anderson K, Tremblay M, Rose CF. AST-120 (spherical carbon adsorbent) lowers ammonia levels and attenuates brain edema in bile duct-ligated rats. Hepatology. 2011;53:1995-2002.

Bosoi, C.R. et al., 2014. Increased brain lactate is central to the development of brain edema in rats with chronic liver disease. Journal of Hepatology, 60(3), p.554-560. 
[16] Davies NA, Wright G, Ytrebø LM, Stadlbauer V, Fuskevåg O-M, Zwingmann C, et al. L-ornithine and phenylacetate synergistically produce sustained reduction in ammonia and brain water in cirrhotic rats. Hepatology. 2009;50:155-164.

[17] Zwingmann C. Nuclear magnetic resonance studies of energy metabolism and glutamine shunt in hepatic encephalopathy and hyperammonemia. J. Neurosci. Res. 2007;85:3429-3442.

[18] Bosoi CR, Yang X, Huynh J, Parent-Robitaille C, Jiang W, Tremblay M, et al. Systemic oxidative stress is implicated in the pathogenesis of brain edema in rats with chronic liver failure. Free Radic. Biol. Med. 2012;52:1228-1235.

[19] Zwingmann C, Chatauret N, Rose C, Leibfritz D, Butterworth RF. Selective alterations of brain osmolytes in acute liver failure: protective effect of mild hypothermia. Brain Res. 2004;999:118-123.

[20] Stacpoole PW, Henderson GN, Yan Z, Cornett R, James MO. Pharmacokinetics, metabolism and toxicology of dichloroacetate. Drug Metab. Rev. 1998;30:499-539.

[21] Bélanger M, Côté J, Butterworth RF. Neurobiological characterization of an azoxymethane mouse model of acute liver failure. Neurochemistry International. 2006;48:434-440.

[22] Marmarou A, Tanaka K, Shulman K. An improved gravimetric measure of cerebral edema. J. Neurosurg. 1982;56:246-253.

[23] Tofteng F, Jorgensen L, Hansen BA, Ott P, Kondrup J, Larsen FS. Cerebral microdialysis in patients with fulminant hepatic failure. Hepatology. 2002;36:1333-1340.

[24] Chatauret N, Rose C, Butterworth RF. Mild hypothermia in the prevention of brain edema in acute liver failure: mechanisms and clinical prospects. Metab Brain Dis. 2002;17:445-451.

[25] Zwingmann C, Chatauret N, Leibfritz D, Butterworth RF. Selective increase of brain lactate synthesis in experimental acute liver failure: results of a [H-C] nuclear magnetic resonance study. Hepatology. 2003;37:420-428.

[26] Chavarria L, Oria M, Romero-Gimenez J, Alonso J, Lope-Piedrafita S, Cordoba J. Diffusion tensor imaging supports the cytotoxic origin of brain edema in a rat model of acute liver failure. Gastroenterology. 2010;138:1566-1573.

[27] Rose C, Ytrebø LM, Davies NA, Sen S, Nedredal GI, Belanger M, et al. Association of reduced extracellular brain ammonia, lactate, and intracranial pressure in pigs with acute liver failure. Hepatology. 2007;46:1883-1892.

[28] Sen S, Rose C, Ytrebø LM, Davies NA, Nedredal GI, Drevland SS, et al. Effect of albumin dialysis on intracranial pressure increase in pigs with acute liver failure: a randomized study. Crit. Care Med. 2006;34:158-164.

[29] Yao H, Sadoshima S, Fujii K, Kusuda K, Ishitsuka T, Tamaki K, et al. Cerebrospinal fluid lactate in patients with hepatic encephalopathy. Eur. Neurol. 1987;27:182-187.

[30] Hindfelt B, Plum F, Duffy TE. Effect of acute ammonia intoxication on cerebral metabolism in rats with portacaval shunts. J. Clin. Invest. 1977;59:386-396.

[31] Therrien G, Giguère JF, Butterworth RF. Increased cerebrospinal fluid lactate reflects deterioration of neurological status in experimental portal-systemic encephalopathy. Metab Brain Dis. 1991;6:225-231.

[32] Hartmann IJ, Groeneweg M, Quero JC, Beijeman SJ, de Man RA, Hop WC, et al. The prognostic significance of subclinical hepatic encephalopathy. Am. J. Gastroenterol. 2000;95:2029-2034.

[33] Lai JC, Cooper AJ. Brain alpha-ketoglutarate dehydrogenase complex: kinetic properties, regional distribution, and effects of inhibitors. J. Neurochem. 1986;47:1376-1386.

[34] Lowry OH, Passonneau JV. Kinetic evidence for multiple binding sites on phosphofructokinase. J. Biol. Chem. 1966;241:22682279.

[35] Fitzpatrick SM, Cooper AJ, Hertz L. Effects of ammonia and beta-methylene-DL-aspartate on the oxidation of glucose and pyruvate by neurons and astrocytes in primary culture. J. Neurochem. 1988;51:1197-1203.

[36] Mans AM, DeJoseph MR, Hawkins RA. Metabolic abnormalities and grade of encephalopathy in acute hepatic failure. J. Neurochem. 1994;63:1829-1838.

[37] Staub F, Baethmann A, Peters J, Weigt H, Kempski O. Effects of lactacidosis on glial cell volume and viability. J. Cereb. Blood Flow Metab. 1990;10:866-876.

[38] Preuss M. An energy-failure based brain edema concept. Med. Hypotheses. 2012;79:259-260.

[39] Martinez-Hernandez A, Bell KP, Norenberg MD. Glutamine synthetase: glial localization in brain. Science. 1977;195:13561358.

[40] Brusilow SW, Traystman R. Hepatic encephalopathy. N. Engl. J. Med. 1986;314:786-787; author reply 787.

[41] Norenberg MD, Bender AS. Astrocyte swelling in liver failure: role of glutamine and benzodiazepines. Acta Neurochir Suppl (Wien). 1994;60:24-27.

[42] Master S, Gottstein J, Blei AT. Cerebral blood flow and the development of ammonia-induced brain edema in rats after portacaval anastomosis. Hepatology. 1999;30:876-880.

[43] Albrecht J, Zielińska M, Norenberg MD. Glutamine as a mediator of ammonia neurotoxicity: A critical appraisal. Biochem. Pharmacol. 2010;80:1303-1308.

[44] Heins J, Zwingmann C. Organic osmolytes in hyponatremia and ammonia toxicity. Metabolic Brain Disease. 2010;25:81-89.

Bosoi, C.R. et al., 2014. Increased brain lactate is central to the development of brain edema in rats with chronic liver disease. Journal of Hepatology, 60(3), p.554-560. 
[45] Cordoba J, Gottstein J, Blei AT. Glutamine, myo-inositol, and organic brain osmolytes after portocaval anastomosis in the rat: implications for ammonia-induced brain edema. Hepatology. 1996;24:919-923.

[46] Cooper AJ, Mora SN, Cruz NF, Gelbard AS. Cerebral ammonia metabolism in hyperammonemic rats. J. Neurochem. 1985;44:1716-1723.

[47] Desjardins P, Rao KV, Michalak A, Rose C, Butterworth RF. Effect of portacaval anastomosis on glutamine synthetase protein and gene expression in brain, liver and skeletal muscle. Metab Brain Dis. 1999;14:273-280.

[48] Kanamori K, Ross BD, Chung JC, Kuo EL. Severity of hyperammonemic encephalopathy correlates with brain ammonia level and saturation of glutamine synthetase in vivo. J. Neurochem. 1996;67:1584-1594.

[49] Schliess F, Görg B, Fischer R, Desjardins P, Bidmon HJ, Herrmann A, et al. Ammonia induces MK-801-sensitive nitration and phosphorylation of protein tyrosine residues in rat astrocytes. FASEB J. 2002;16:739-741.

[50] Kamiya T, Katayama Y, Kashiwagi F, Terashi A. The role of bradykinin in mediating ischemic brain edema in rats. Stroke. 1993;24:571-575; discussion 575-576.

[51] Nonaka M, Yoshimine T, Kohmura E, Wakayama A, Yamashita T, Hayakawa T. Changes in brain organic osmolytes in experimental cerebral ischemia. J. Neurol. Sci. 1998;157:25-30.

[52] Van der Zijden JP, van Eijsden P, de Graaf RA, Dijkhuizen RM. 1H/13C MR spectroscopic imaging of regionally specific metabolic alterations after experimental stroke. Brain. 2008;131:2209-2219.

[53] Cauli O, López-Larrubia P, Rodrigo R, Agusti A, Boix J, Nieto-Charques L, et al. Brain region-selective mechanisms contribute to the progression of cerebral alterations in acute liver failure in rats. Gastroenterology. 2011;140:638-645.

[54] Rose CF. Ammonia-lowering strategies for the treatment of hepatic encephalopathy. Clin. Pharmacol. Ther. 2012;92:321-331.

[55] Abdelmalak M, Lew A, Ramezani R, Shroads AL, Coats BS, Langaee T, et al. Long-term safety of dichloroacetate in congenital lactic acidosis. Mol. Genet. Metab. 2013;109:139-143.

[56] Strum SB, Adalsteinsson O, Black RR, Segal D, Peress NL, Waldenfels J. Case report: Sodium dichloroacetate (DCA) inhibition of the "Warburg Effect" in a human cancer patient: complete response in non-Hodgkin's lymphoma after disease progression with rituximab-CHOP. J. Bioenerg. Biomembr. 2013;45:307-315.

[57] Calvert LD, Shelley R, Singh SJ, Greenhaff PL, Bankart J, Morgan MD, et al. Dichloroacetate enhances performance and reduces blood lactate during maximal cycle exercise in chronic obstructive pulmonary disease. Am. J. Respir. Crit. Care Med. 2008;177:1090-1094.

Bosoi, C.R. et al., 2014. Increased brain lactate is central to the development of brain edema in rats with chronic liver disease. Journal of Hepatology, 60(3), p.554-560. 